\title{
Throughput and Delay Analysis of Wireless Multicast in Distributed Mobile Social Networks Based on Geographic Social Relationships
}

\author{
Jie Hu, Lie-Liang Yang, and Lajos Hanzo \\ School of Electronics and Computer Science, University of Southampton, Southampton, UK, SO17 1BJ, \\ Tel: +44-23-8059 3125, Fax: +44-23-8059 4508 \\ Email: \{jh10g11, lly, lh\}@ecs.soton.ac.uk, http://www-mobile.ecs.soton.ac.uk
}

\begin{abstract}
Since mobile communications exhibit strong social characteristics, based on the potential common interests of mobile users, mobile social networks (MSNs) are capable of mitigating the tele-traffic bottle-neck. By multicasting the content of common interest from a content owner to content seekers within the owner's transmission range, a distributed MSNs architecture is proposed, which is capable of mitigating the tele-traffic imposed on network operators. In this contribution, the social relationship between a pair of MSN users is defined according to their geographic characteristics. By jointly considering the geographic social relationships and the wireless propagation environment, we derive the closed-form equations for evaluating both the throughput and delay of the social unicast/multicast transmissions. Simulation results are provided, both for supporting our theoretical analysis, as well as for investigating the impact of social relationships on the achievable network performance. Based on the results presented, we conclude that a more sociallyminded content owner is particularly efficient in multicasting the content of common interest to content seekers.
\end{abstract}

\section{INTRODUCTION}

The design of Mobile Social Networks (MSNs) [1] is governed by the combination of mobile networking and social science principles, and in recent years they have attracted much attention in both the industrial and academic communities. There are two types of MSNs, namely centralized MSNs and distributed MSNs [2]. In centralized MSNs, such as Facebook accessed from smart mobile phones, both the social relationships of MSN users and the contents of common interest are stored in centralized online servers. The interaction between a pair of MSN users is established through the centralized infrastructure, such as the base stations (BSs) and WIFI hotspots, for example. By contrast, in distributed MSNs, such as EyeVibe [3], the social relationships of MSN users have to be discovered on a case-by-case basis. Based on these social relationships, a self-organized ad hoc network is constructed for disseminating the content of common interest from content owners to content seekers. Distributed MSNs are thus capable of reducing the tele-traffic imposed on the BSaided architecture of wireless network operators.

The financial support of the RC-UK's India-UK Advanced Technology Centre (IU-ATC), that of the EU's concerto project, that of the EU's Advanced Fellow Grant and that of the China Scholarship Council (CSC) is gratefully acknowledged.

This paper is going to be published in the proceedings of IEEE Wireless Communications and Networking Conference (WCNC) 2014.
In recent years, substantial advances have been made in the area of MSNs. The social relationships amongst the MSN users were represented in terms of the definitions of betweenness and similarity ${ }^{1}$ in graph theory, which were exploited for designing the routing protocol referred to as SimBet [4]. In [5], the authors exploited the communication history between a pair of users for updating their social relationship. The authors of [6] derived a tight theoretical upper bound of the 'flooding' time, which was defined as the number of time-steps required for broadcasting a message from a source node to every node in mobile ad hoc networks.

Although the research reported in the above-mentioned contributions is in line with the concept of distributed MSNs, the authors of [4] - [6] did not propose a practical architecture for distributed MSNs. Moreover, the results of [4] - [6] relied on the users' mobility patterns, while the impact of the wireless propagation environment was to a large extent ignored. Furthermore, as claimed in [7], two thirds of social relationships are determined by the users' geographic locations, while in Kleinberg's work [8], the probability of whether a social relationship exists or not is determined by the geographic distance between a pair of users. Based on the same definition of the social relationships, the authors of [9] explored the attainable network capacity by considering the impact of geographic social relationships. However, both the achievable throughput and the delay imposed by wireless multicast invoked in a distributed MSN architecture have remained hitherto largely unexplored, especially in the light of the geographic social relationships and of the specific wireless propagation scenarios. Hence, we devote particular attention to these design aspects.

More specifically, in order to fill this gap, our paper has the following novel contributions:

(1) An architecture is proposed for distributed MSNs.

(2) The neighbourhood range, regular contacts and opportunistic contacts, as well as the social strength are defined for describing the geographic social relationships within the distributed MSNs considered.

(3) The statistical properties of both the throughput and the delay of the social unicast/multicast are derived by considering both the geographic social relationships and the diverse

${ }^{1}$ Similarity is calculated based on the number of common neighbours of each node. 


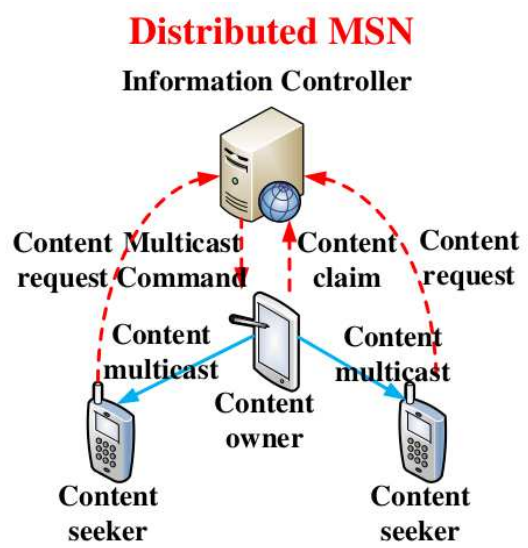

Fig. 1: Distributed MSN architecture wireless propagation scenarios.

In the rest of this paper, we first outline our system model in Section II. Then both the relevant throughput and delay performances are analysed theoretically in Section III, while our numerical results are provided in Section IV for the sake of supporting our theoretical analysis. Finally, we conclude the paper in Section V.

\section{System Model}

\section{A. Social Multicast in Distributed MSN}

Let us continue by considering the scenario as shown in Fig.1. After recording a piece of video clip, a MSN user (content owner) sends a message to the information controller to claim the authorship of the video clip. After receiving this message, the information controller broadcasts it to all the MSN users. Any MSN users (content seekers), who are interested in the specific video clip considered would report their interests to the information controller. By synthesizing all the information collected from the MSN users, a temporary community can be established, which includes the content owner and all the content seekers within the content owner's transmission range. Then, the constitution of the community is broadcast to all of its members. As a result, an ad hoc network is created by self-organization, based on which the video clip is multicast simultaneously from the content owner to the content seekers. Finally, once all the content seekers are satisfied, the community may be dismissed.

\section{B. Geographic Social Relationship}

We assume that a pair of MSN users may share a social relationship with a probability of $\varphi$, which is also termed as the social strength that is determined by the geographic distance of $Y=y$ between this pair of MSN users. As shown in Fig.2, there are two types of contacts for a content owner, namely regular contacts and opportunistic contacts. Content seekers within the content owner's neighbourhood range $r$ form his/her regular contacts, which are established with a probability of $\varphi=1$. By contrast, content seekers beyond the content owner's neighbourhood range $r$ form his/her opportunistic contacts. These opportunistic contacts are established with a specific probability of $\varphi$, which is inversely proportional to $Y=y$ with

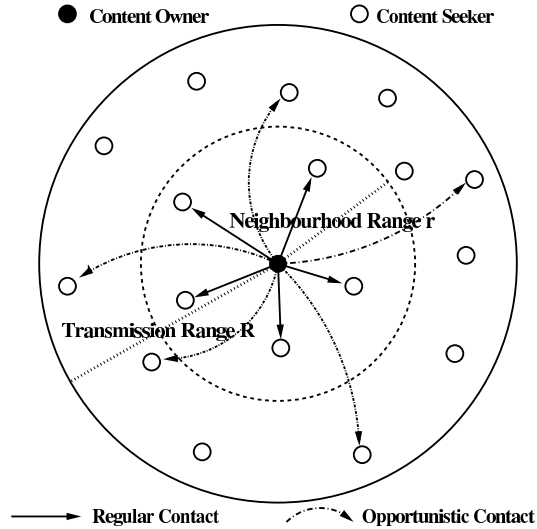

Fig. 2: Geographic social relationships and characteristics

the exponent of $\alpha$ [7]. Thus, given a specific distance $Y=y$, the social strength $\varphi$ is defined as

$$
\left.\varphi\right|_{Y=y}=\left\{\begin{array}{l}
1,0 \leq y \leq r, \\
\frac{1}{(y / r)^{\alpha}}, y>r,
\end{array}\right.
$$

where the exponent $\alpha$ is termed as the social exponent. We assume that the geographic social relationship between a pair of MSN users does not change during a time slot (TS), but varies independently from one TS to another.

\section{Geographic Characteristics}

We assume that all the content seekers roam in a circular area having a radius ${ }^{2}$ of $R$, while the content owner is stationary at the center of the circular area, as shown in Fig.2. In line with the mobility model introduced in [10], the position of the $i$ th MSN user during the $t$ th TS is defined as $\mathbf{P}_{i}(t)$, which may be modelled by a stationary and ergodic process having a stationary uniform distribution. Moreover, the positions of different MSN users are independent and identically distributed (i.i.d.). Hence, the CDF and PDF of the random distance $Y$ between a content owner and content seeker pair are formulated as:

$$
F_{Y}(y)=\frac{y^{2}}{R^{2}}, f_{Y}(y)=\frac{d F_{Y}(y)}{d y}=\frac{2 y}{R^{2}}, 0 \leq y \leq R .
$$

\section{PHY Layer}

In the PHY layer, the wireless link delivering the content from a content owner to a content seeker is attenuated by path loss (PL) and additionally, it is subjected to small-scale fading.

1) Small-scale fading: The small-scale radio propagation phenomenon is modelled by uncorrelated stationary Rayleigh flat-fading. The channel's amplitude $|h(t)|$, which varies from one TS to another, is a Rayleigh distributed random variable during the $t$ th TS. Consequently, the square of the channel amplitude $|h(t)|^{2}$ obeys an exponential distribution associated with $E\left[|h(t)|^{2}\right]=1$. The PDF and CDF of $X=|h(t)|^{2}$ are $f_{X}(x)=\exp (-x), F_{X}(x)=1-\exp (-x), x>0$, respectively.

\footnotetext{
${ }^{2}$ This radius is also the transmission range of the content owner
} 
2) Path loss (PL): According to [11], the PL equation is invalid for calculating the attenuation in the near-field of the transmit antennas. Thus, we assume that the PL only imposes attenuation on a wireless link, if its distance is longer than a reference threshold $d_{0}$. Hence, the PL model is formulated as

$$
\frac{P_{r}}{P_{0}}= \begin{cases}1, & 0 \leq y \leq d_{0}, \\ \frac{1}{\left(y / d_{0}\right)^{\kappa}}, & y>d_{0}\end{cases}
$$

where $P_{0}$ is the reference power received at $d_{0}$, which may be deemed identical to the transmit power of the content owner. Furthermore, $P_{r}$ is the power received at the content seeker after being attenuated by the PL, while $\kappa$ is the PL exponent.

\section{E. The Successful Packet Delivery Probability}

A packet of common interest can be successfully delivered from a content owner to a content seeker during a TS, if the following two events occur simultaneously. Firstly, a social relationship must exist between a pair of content owner and seeker, which is determined by the social strength $\varphi$ of (1), and secondly, despite being attenuated by the PL and small-scale fading, the instantaneous received SNR at a content seeker must exceed a predefined SNR threshold $\gamma$.

Provided that the PHY layer obeys the model introduced in Section II-D, the instantaneous received SNR is $P_{0}|h(t)|^{2} /\left(N_{0} W\right)$ if the transmission distance $y$ is shorter than $d_{0}$, while the instantaneously received SNR is $P_{0}|h(t)|^{2} /\left(y / d_{0}\right)^{K} /\left(N_{0} W\right)$ if the transmission distance $y$ is longer than $d_{0}$. Here, $N_{0} W$ is the power of the white Gaussian noise. As a result, given the transmission distance $Y=y$ and the social strength defined in (1), the successful packet delivery probability of a social wireless link connecting a content owner to a content seeker during a TS may be expressed as

$\left.\mu\right|_{Y=y}= \begin{cases}\left.P\left(|h(t)|^{2}>A d_{0}^{K}\right) \varphi\right|_{Y=y}=\left.\exp \left(-A d_{0}^{K}\right) \varphi\right|_{Y=y}, & 0 \leq y \leq d_{0}, \\ \left.P\left(|h(t)|^{2}>A y^{K}\right) \varphi\right|_{Y=y}=\left.\exp \left(-A y^{K}\right) \varphi\right|_{Y=y}, & y>d_{0},\end{cases}$

where $A=\left(\gamma N_{0} W\right) /\left(P_{0} d_{0}^{\kappa}\right)$. Naturally, $\mu$ is equivalent to the normalized throughput of the social unicast, which has a unit of packets/TS [12].

Moreover, automatic repeat request (ARQ) relying on an unlimited number of re-transmissions may be adopted for ensuring that no packets of the contents are lost.

\section{Throughrut and Delay Analysis}

In this section, we derive the statistical properties of both the throughput and the delay in the social unicast/multicast transmission scenarios considered.

\section{A. The Social Unicast Transmission}

1) The social unicast throughput: When jointly considering both the PL of (3) and the social strength of (1), we may have different expressions for $\left.\mu\right|_{Y=y}$, depending on the neighbourhood range $r$ and on the PL reference distance $d_{0}$. In order to derive the $m$ th moment $E\left[\mu^{m}\right]$ of the social unicast throughput, we may integrate $\left.\mu^{m}\right|_{Y=y}$ over the PDF of the distance $Y=y$ between the content owner and the content receiver, which is given by (2). The following two cases are considered.
Case 1: If $d_{0} \leq r$, then we have the following expression of $\left.\mu_{(1)}^{m}\right|_{Y=y}$ :

$$
\mu_{(1)}^{m} \mid \boldsymbol{Y}=y= \begin{cases}\exp \left(-m A d_{0}^{K}\right), & 0 \leq y \leq d_{0}, \\ \exp \left(-m A y^{K}\right), & d_{0}<y \leq r, \\ \frac{\exp \left(-m A y^{K}\right)}{(y / r)^{m \alpha}}, & r<y \leq R\end{cases}
$$

As a result, we may derive the $m$ th moment of the social unicast throughput as

$$
\begin{aligned}
E\left[\mu_{(1)}^{m}\right] & =\int_{0}^{R}\left(\left.\mu_{(1)}^{m}\right|_{Y=y}\right) f_{Y}(y) d y, \\
& =\underbrace{\int_{0}^{d_{0}} \exp \left(-m A d_{0}^{K}\right) \frac{2 y}{R^{2}} d y}_{I_{1}^{(1)}}+\underbrace{\int_{d_{0}}^{r} \exp \left(-m A y^{K}\right) \frac{2 y}{R^{2}} d y}_{I_{2}^{(1)}} \\
& +\underbrace{\int_{r}^{R} \exp \left(-m A y^{K}\right)\left(\frac{r}{y}\right)^{m \alpha} \frac{2 y}{R^{2}} d y}_{I_{3}^{(1)}},
\end{aligned}
$$

where the second equality is obtained by substituting (2) into the first line of (6). Hence, the first integral is obtained as

$$
I_{1}^{(1)}=\int_{0}^{d_{0}} \exp \left(-m A d_{0}^{K}\right) \frac{2 y}{R^{2}} d y=\left.\exp \left(-m A d_{0}^{K}\right) \frac{y^{2}}{R^{2}}\right|_{0} ^{d_{0}},
$$

while the second integral is derived as

$$
I_{2}^{(1)}=\frac{2}{R^{2}} \int_{d_{0}}^{r} \exp \left(-m A y^{K}\right) y d y=\left.\frac{2}{R^{2}} \Phi(y \mid 1,0, m A)\right|_{d_{0}} ^{r},
$$

where the function $\Phi(y \mid \beta, \alpha, A)$ is defined as

$$
\begin{aligned}
& \Phi(y \mid \beta, \alpha, A)=\int y^{\beta-\alpha} \exp \left(-A y^{K}\right) d y \\
& = \begin{cases}-\frac{A^{z_{1}} \Gamma\left(-z_{1}, A y^{\kappa}\right)}{\kappa}, & z_{1}=\frac{\alpha-\beta-1}{\kappa}, \\
-\frac{\Gamma\left(z_{2}, A y^{K}\right)}{\kappa A^{z_{2}}}, & \text { if } \beta<\alpha, \\
z_{2}=\frac{\beta-\alpha+1}{\kappa}, & \text { if } \beta \geq \alpha,\end{cases}
\end{aligned}
$$

while the functions $\Gamma\left(-z_{1}, A y^{K}\right)$ and $\Gamma\left(z_{2}, A y^{\kappa}\right)$ are given by the following equations [13]:

$$
\begin{gathered}
\Gamma\left(-z_{1}, A y^{K}\right)=\int_{A y^{\kappa}}^{\infty} \frac{1}{z^{z_{1}+1}} \exp (-t) d t, \\
\Gamma\left(z_{2}, A y^{K}\right)=\int_{A y^{\kappa}}^{\infty} t^{z_{2}-1} \exp (-t) d t .
\end{gathered}
$$

In a similar way, we may derive the third integral in (6) as

$I_{3}^{(1)}=\frac{2 r^{m \alpha}}{R^{2}} \int_{r}^{R} \exp \left(-m A y^{K}\right) y^{1-m \alpha} d y=\left.\frac{2 r^{m \alpha}}{R^{2}} \Phi(y \mid 1, m \alpha, m A)\right|_{r} ^{R}$.

Finally, we may arrived at the closed-form expression of the $m$ th moment of the social unicast throughput, when $d_{0} \leq r$, which is $E\left[\mu_{(1)}^{m}\right]=I_{1}^{(1)}+I_{2}^{(1)}+I_{3}^{(1)}$.

Case 2: If $d_{0}>r$, then we have the following expression of $\left.\mu_{(2)}^{m}\right|_{Y=y}$ :

$$
\mu_{(2)}^{m} \mid \boldsymbol{Y}=y= \begin{cases}\frac{\exp \left(-m A d_{0}^{\kappa}\right),}{\exp \left(-m A d_{0}^{\kappa}\right)} & 0 \leq y \leq r, \\ \frac{(y / r)^{m \alpha}}{\exp \left(-m A y^{K}\right)} & r<y \leq d_{0}, \\ (y / r)^{m \alpha} & d_{0}<y \leq R\end{cases}
$$


As a result, we may derive the $m$ th moment of the social unicast throughput as

$$
\begin{aligned}
E\left[\mu_{(2)}^{m}\right] & =\int_{0}^{R}\left(\left.\mu_{(2)}^{m}\right|_{Y=y}\right) f_{Y}(y) d y, \\
& =\underbrace{\int_{0}^{r} \exp \left(-m A d_{0}^{K}\right) \frac{2 y}{R^{2}} d y}_{I_{1}^{(2)}}+\underbrace{\int_{r}^{d_{0}} \exp \left(-m A d_{0}^{K}\right)\left(\frac{r}{y}\right)^{m \alpha} \frac{2 y}{R^{2}} d y}_{I_{2}^{(2)}} \\
& +\underbrace{\int_{d_{0}}^{R} \exp \left(-m A y^{K}\right)\left(\frac{r}{y}\right)^{m \alpha} \frac{2 y}{R^{2}} d y}_{I_{3}^{(2)}},
\end{aligned}
$$

where the second equality is obtained by substituting (2) into the first line of (9). Hence, the first integral is obtained as

$$
I_{1}^{(2)}=\int_{0}^{r} \exp \left(-m A d_{0}^{K}\right) \frac{2 y}{R^{2}} d y=\left.\exp \left(-m A d_{0}^{K}\right) \frac{y^{2}}{R^{2}}\right|_{0} ^{r},
$$

while the second integral is derived as

$$
\begin{aligned}
I_{2}^{(2)} & =\frac{2 r^{m \alpha}}{R^{2}} \exp \left(-m A d_{0}^{K}\right) \int_{r}^{d_{0}} y^{1-m \alpha} d y \\
& =\left.\frac{2 r^{m \alpha}}{R^{2}} \exp \left(-m A d_{0}^{K}\right) \Psi(y \mid 1, m \alpha)\right|_{r} ^{d_{0}},
\end{aligned}
$$

where the function $\Psi(y \mid \beta, \alpha)$ is defined as

$$
\Psi(y \mid \beta, \alpha)=\int y^{\beta-\alpha} d y= \begin{cases}\frac{y^{\beta-\alpha+1}}{\beta-\alpha+1}, & \text { if } \beta-\alpha \neq-1, \\ \ln y, & \text { if } \beta-\alpha=-1 .\end{cases}
$$

In a similar way, we may derive the third integral in (9) as

$$
I_{3}^{(2)}=\frac{2 r^{m \alpha}}{R^{2}} \int_{d_{0}}^{R} \exp \left(-m A y^{\kappa}\right) y^{1-m \alpha} d y=\left.\frac{2 r^{m \alpha}}{R^{2}} \Phi(y \mid 1, m \alpha, m A)\right|_{d_{0}} ^{R} .
$$

Finally, we may arrive at the closed-form expression of the $m$ th moment of the social unicast throughput, when $d_{0}>r$, which is $E\left[\mu_{(2)}^{m}\right]=I_{1}^{(2)}+I_{2}^{(2)}+I_{3}^{(2)}$.

Consequently, according to the expression of the $m$ th moment of the social unicast throughput, we arrive at its average $E[\mu]$ by setting $m=1$, as well as its variance $\operatorname{Var}[\mu]=$ $E\left[\mu^{2}\right]-\{E[\mu]\}^{2}$ by setting $m=2$ for deriving the second moment of the social unicast throughput.

2) The social unicast delay: Another significant performance metric is the social unicast delay of $K_{i}$ TSs from the content owner to the $i$ th content seeker, which indicates that the content seeker $i$ first successfully receives the packet during the $K_{i}$ th TS. Since the successful packet reception probability $\mu_{i}$ of the content seeker $i$ varies from one TS to another, which is caused by the time-varying distance between the content owner and the content seeker $i$, it is hard for us to derive the exact CDF and probability mass function (PMF) for $K_{i}$. However, if we approximate the time-varying $\mu_{i}$ by its average value of $E\left[\mu_{i}\right]=E[\mu]$ derived above, we note that the social unicast delay $K_{i}$ obeys a geometric distribution associated with a success probability of $E[\mu]$. Consequently, the approximated PMF and the CDF of $K_{i}$ are

$$
P\left(K_{i}=k_{i}\right) \approx(1-E[\mu])^{k_{i}-1} \cdot E[\mu], k_{i} \geq 1,
$$

$$
P\left(K_{i} \leq k_{i}\right) \approx \sum_{n=1}^{k_{i}} P\left(K_{i}=n\right)=1-(1-E[\mu])^{k_{i}}, k_{i} \geq 1,
$$

respectively. Hence the average value of $K_{i}$ is derived as

$$
E\left[K_{i}\right] \approx \sum_{n=1}^{+\infty} n \cdot P\left(K_{i}=n\right)=\frac{1}{E[\mu]}
$$

The accuracy of our approximation will be validated by the simulation results provided in Section IV.

\section{B. The Social Multicast Transmission}

1) The social multicast delay: Let us now derive the social multicast delay of $K_{m u l}$ TSs, which is defined as the time when all the content seekers within the transmission range of the content owner receive the desired packet. We assume that there are $N$ content seekers within the transmission range of the content owner. The social multicast delay $K_{m u l}$ is defined as $K_{m u l}=\max \left\{K_{1}, K_{2}, \ldots, K_{i}, \ldots, K_{N}\right\}$, where $K_{i}$ is the social unicast delay of the content seeker $i$. Hence, the CDF of the social multicast delay $K_{m u l}$ is derived as

$$
P\left(K_{\text {mul }} \leq k_{\text {mul }}\right)=P\left(\max \left\{K_{1}, K_{2}, \ldots, K_{i}, \ldots, K_{N}\right\} \leq k_{m u l}\right),
$$

Since $\left\{K_{i}, i=1,2, \ldots, N\right\}$ are i.i.d. random variables, we may derive the CDF of $K_{m u l}$ as

$$
\begin{aligned}
P\left(K_{m u l} \leq k_{m u l}\right) & =P\left(K_{1} \leq k_{m u l}\right) \cdot P\left(K_{2} \leq k_{m u l}\right) \cdot \ldots \cdot P\left(K_{N} \leq k_{m u l}\right), \\
& \approx\left\{1-(1-E[\mu])^{k_{m u l}}\right\}^{N}, k_{m u l} \geq 1
\end{aligned}
$$

where the second approximate equality is obtained by exploiting the approximation made in (12). As a result, we may express the PMF of $K_{m u l}$ as

$$
\begin{aligned}
P\left(K_{m u l}=k_{m u l}\right) & =P\left(K_{m u l} \leq k_{m u l}\right)-P\left(K_{m u l} \leq k_{m u l}-1\right) \\
& \approx\left\{1-(1-E[\mu])^{k_{m u l}}\right\}^{N}-\left\{1-(1-E[\mu])^{k_{m u l}-1}\right\}^{N} .
\end{aligned}
$$

Furthermore, the average social multicast delay is formulated as

$$
E\left[K_{m u l}\right]=\sum_{k_{m u l}=1}^{\infty} k_{m u l} P\left(K_{m u l}=k_{m u l}\right),
$$

where $P\left(K_{m u l}=k_{m u l}\right)$ is given by (16).

2) The social multicast throughput: Since each of the $N$ content seekers receives a copy of the packet at the end of the multicast session in the distributed MSN considered, $N$ copies of the packet are transmitted during the multicast process. As a result, the multicast regime's throughput $\mu_{m u l}$ is determined by the number of content seekers within the content owner's transmission range as well as by the social multicast delay $K_{m u l}$, which can be expressed as $\mu_{m u l}=N / K_{m u l}$ packets $/ \mathrm{TS}$. Hence, the $m$ th moment of the content owner's social multicast throughput is formulated as

$$
E\left[\mu_{m u l}^{m}\right]=E\left[\left(\frac{N}{K_{m u l}}\right)^{m}\right]=\sum_{k_{m u l}=0}^{\infty}\left(\frac{N}{k_{m u l}}\right)^{m} P\left(K_{m u l}=k_{m u l}\right),
$$

where $P\left(K_{m u l}=k_{m u l}\right)$ is given by (16). As a result, by letting $m=1$ in (18), we arrive at the content owner's average social multicast throughput. Moreover, we may express its variance by letting $m=2$, which is $\operatorname{Var}\left(\mu_{m u l}\right)=E\left[\mu_{m u l}^{2}\right]-E\left[\mu_{m u l}\right]^{2}$. 

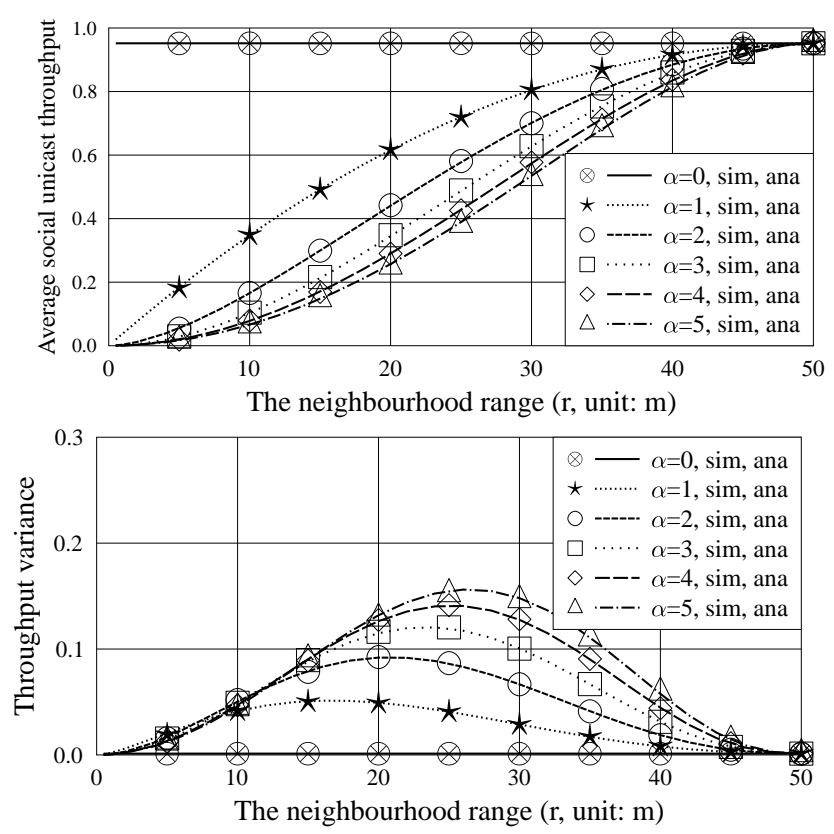

Fig. 3: Statistical properties of the social unicast throughput.

\section{Numerical Results}

In the PHY layer, we set the reference distance of the PL model to be $d_{0}=1$ meter $(\mathrm{m})$. The transmit power is set to $P_{0}=-24 \mathrm{dBm}$, while the noise power is $N_{0} W=-94 \mathrm{dBm}$. The PL exponent is set to $\kappa=3$, while the successful packet reception SNR threshold is $\gamma=10 \mathrm{~dB}$. Moreover, we assume that all the content seekers roam in the circular area constituted by the content owner's transmission range associated with a radius of $R=50 \mathrm{~m}$, and the content owner is at the center of this area. We run the simulations 100000 times in Matlab for the sake of accurately characterizing the associated statistical properties.

\section{A. The Social Unicast Transmission}

As shown in the top trace of Fig.3, the average social unicast throughput increases as we increase the neighbourhood range $r$ of the content owner, provided that the social exponent $\alpha$ is not zero. A higher value of the neighbourhood range $r$ indicates that a content seeker is more likely to be one of the content owner's regular contacts, which substantially enhances the social unicast throughput. However, a higher social exponent $\alpha$ reduces the attainable throughput. This is because, as shown in (1), a higher $\alpha$ indicates that the content owner is more likely to communicate with his/her neighbourhood. Hence a content seeker roaming outside the neighbourhood range is less likely to receive the packet from the content owner. Moreover, when $r$ approaches the transmission range of $R=50 \mathrm{~m}$, which indicates that all the content seekers within the transmission range are regular contacts of the content owner, the throughput approaches its maximum value. In contrast to all other cases, $\alpha=0$ indicates that the opportunistic contacts of the content owner are equivalent to his/her regular contacts. Therefore, the average social unicast throughput, characterised by the top trace of Fig.3, is no longer affected by the content owner's neighbourhood range. In this case, an upper bound is provided

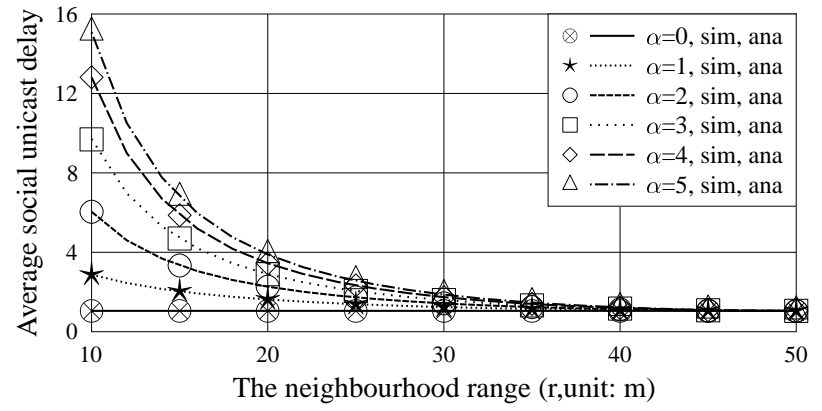

Fig. 4: The average social unicast delay

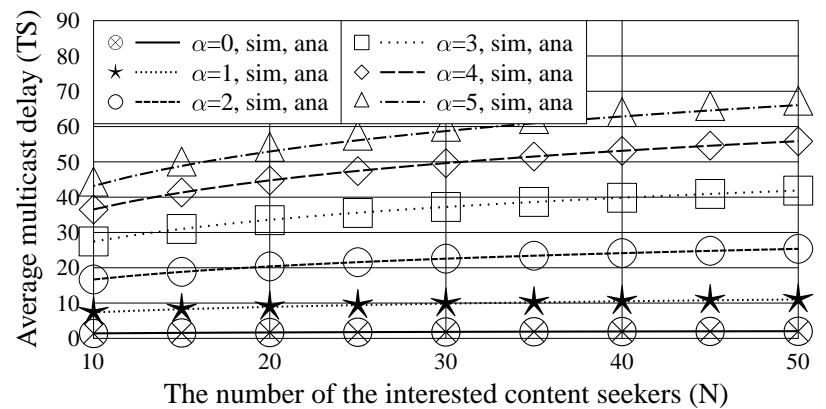

Fig. 5: The average social multicast delay

for the average social unicast throughput, which is equivalent to that of the scenario of $r=R=50 \mathrm{~m}$.

As shown in the bottom trace of Fig.3, when the neighbourhood range $r$ is low or the social exponent $\alpha$ is high, the social strength between a content seeker and the content owner, defined in (1), becomes weak, which results in a low social unicast throughput. Hence, its variance is also quite low. As $r$ gradually increases, a content seeker tends to acquire a higher chance of becoming the content owner's regular contact. This closer relationship hence tends to increase the probability of having an increased social unicast throughput. As a result, the variance gradually becomes higher and approaches its peak. If the neighbourhood range $r$ continues to increase, for example, beyond $25 \mathrm{~m}$ associated with $\alpha=5$, a content seeker tends to have a gradually reduced chance of becoming the content owner's opportunistic contact, which results in a reduced probability of having a low throughput. Therefore, the variance starts to decay in the bottom trace of Fig.3. The simulation (sim) results presented in Fig. 3 validate our throughput analysis (ana) provided in Section III-A.

As portrayed in Fig.4, the social unicast delay reduces towards its lower bound of a single TS, as the neighbourhood range $R$ gradually increases from $10 \mathrm{~m}$ to $50 \mathrm{~m}$. Furthermore, a higher social exponent $\alpha$ also leads to a higher delay for the same reason as argued in the context of Fig.3. Moreover, the accuracy of the approximation made in (11) is also confirmed by the simulation results of Fig. 4 .

\section{B. The Social Multicast Transmission}

It may be readily observed from Fig. 5 that the average social multicast delay is increased, as we increase the total number of content seekers from 10 to 50 within the transmission range of the content owner. Additionally, a higher social exponent $\alpha$, which indicates that the content owner is more reluctant to multicast the content to the content seekers outside the 


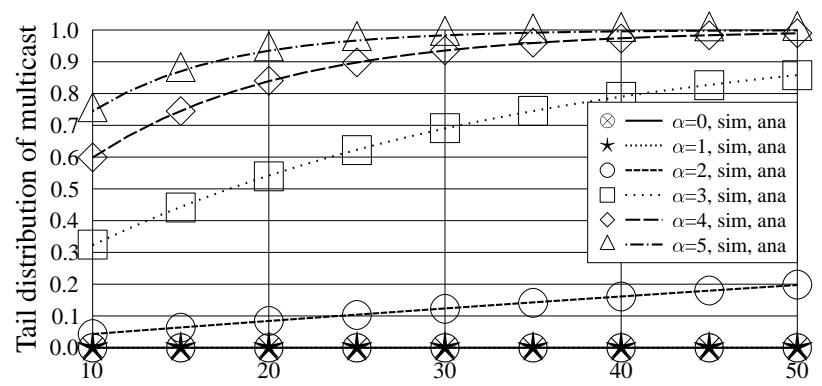

The number of the interested content seekers $(\mathrm{N})$

Fig. 6: The tail distribution of the social multicast delay

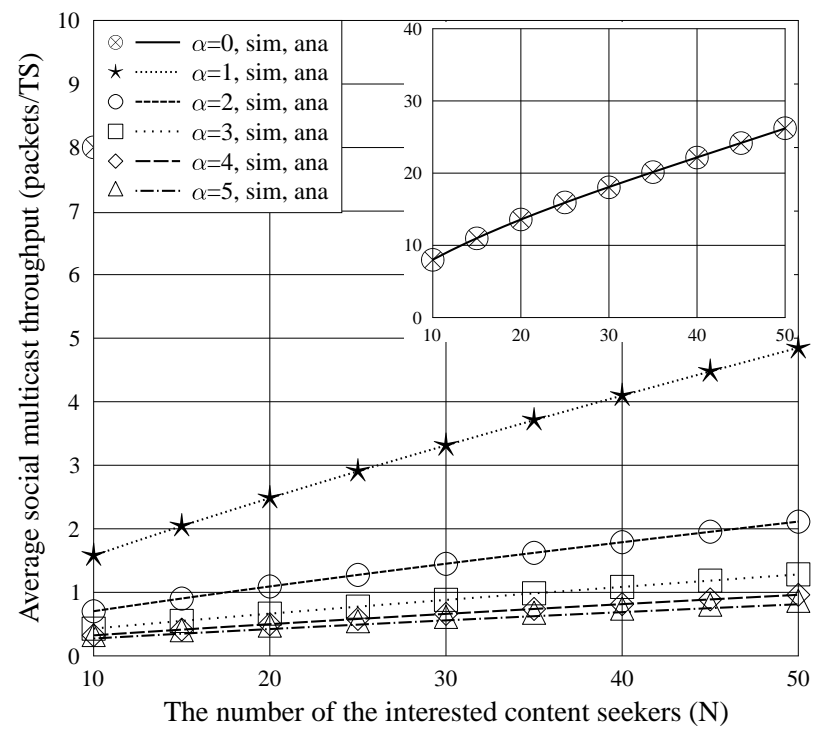

Fig. 7: The average social multicast throughput

neighbourhood range, imposes an increased average social multicast delay. Finally, our simulation (sim) results of Fig.5 confirmed the accuracy of our theoretical analysis (ana) provided in Section III-B.

In order to investigate the tail distribution of the social multicast delay, we set the delay threshold to be 30 TSs. As shown in Fig.6, the probability of the multicast delay exceeding $30 \mathrm{TSs}$ increases as we increase the number of content seekers within the content owner's transmission range. Additionally, the social multicast suffers from a higher tail probability in case of a higher social exponent $\alpha$. For example, when supporting 10 content seekers, the tail probability is around $76 \%$ for $\alpha=5$, while it is only $5 \%$ for $\alpha=2$. Furthermore, the accuracy of our approximation invoked in (14) of Section III-B is confirmed by the simulation results of Fig.6.

It may be observed from Fig.7 that the average social multicast throughput defined in Section III-B increases steadily, as the number of content seekers increases, while a lower social exponent $\alpha$ leads to an improved average social multicast throughput. However, the improvement of the social multicast throughput becomes more limited for a higher $\alpha$, as we increase the number of the content seekers. For example, for $\alpha=5$, the average social multicast throughput is only improved by about 0.5 packets/TS, when the number of the content seekers increases from 10 to 50 .

Hence, based on the results presented in this section, we may claim that a more socially-minded content owner, who has a higher neighbourhood range $r$ and a lower social exponent $\alpha$, is capable of substantially improving the efficiency of the social multicast to the content seekers.

\section{Conclusion}

A distributed MSN architecture was proposed. By jointly considering both the geographic social relationships, where content seekers are categorized into regular and opportunistic contacts of the content owner, as well as the wireless propagation environment, we studied the multicast process facilitated by the content owner for distributing the content of common interest to the content seekers within his/her transmission range. We derived the $m$ th moment of the social unicast/multicast throughput, as well as the approximated PMF and $\mathrm{CDF}$ of the social unicast/multicast delay. Based on the numerical results, we clearly interpreted the impact of the socialrelationships-related parameters on both the performance of the social unicast/multicast. If a content owner has more regular contacts, which is represented by an increased neighbourhood range, the attainable throughput is enhanced and the delay is reduced for the social unicast/multicast scenarios considered. By contrast, if the communications are more likely to be confined to the content owner's neighbourhood range, which is represented by an increased social exponent $\alpha$, the attainable throughput is reduced and the delay is increased.

\section{REFERENCES}

[1] N. Kayastha, D. Niyato, P. Wang, and E. Hossain, "Applications, architectures, and protocol design issues for mobile social networks: A survey," Proceedings of the IEEE, vol. 99, no. 12, pp. $2130-2158$, December 2011.

[2] J. HU, L.-L. YANG, and L. Hanzo, "Mobile social networking aided content dissemination in heterogeneous networks," China Communications, vol. 10, no. 6, pp. 1-13, 2013. [Online]. Available: http://www.chinacommunications.cn/EN/abstract/article_8138.shtml

[3] EyeVibe. [Online]. Available: http://www.eyevibe.net/faq.html

[4] E. Daly and M. Haahr, "Social network analysis for information flow in disconnected delay-tolerant manets," IEEE Transactions on Mobile Computing, vol. 8, no. 5, pp. 606 -621, may 2009.

[5] E. Bulut and B. Szymanski, "Exploiting friendship relations for efficient routing in mobile social networks," IEEE Transactions on Parallel and Distributed Systems, vol. 23, no. 12, pp. 2254 -2265, dec. 2012.

[6] A. Clementi, F. Pasquale, and R. Silvestri, "Opportunistic MANETs: Mobility can make up for low transmission power," IEEE/ACM Transactions on Networking, vol. PP, no. 99, p. 1, 2012.

[7] D. Liben-Nowell, J. Novak, R. Kumar, P. Raghavan, and A. Tomkins, "Geographic routing in social networks," Proceedings of the National Academy of Sciences of the United States of America, vol. 102, no. 33, pp. $11623-11628$, Aug. 2005.

[8] J. Kleinberg, "The small-world phenomenon: an algorithm perspective," in Proceedings of the thirty-second annual ACM symposium on Theory of computing, ser. STOC '00. New York, NY, USA: ACM, 2000, pp. $163-170$.

[9] B. Azimdoost, H. Sadjadpour, and J. Garcia-Luna-Aceves, "The impact of social groups on the capacity of wireless networks," 2011 IEEE Network Science Workshop (NSW), pp. 30 -37, June 2011.

[10] M. Grossglauser and D. Tse, "Mobility increases the capacity of ad hoc wireless networks," IEEE/ACM Transactions on Networking, vol. 10, no. 4, pp. 477-486, 2002.

[11] T. Rappaport, Wireless Communications: Principles and Practice, 2nd ed. Upper Saddle River, NJ, USA: Prentice Hall PTR, 2001.

[12] L. Hanzo, L. Yang, and J. Hu, "Queue scheduling and performance analysis of delay-constrained hybrid cognitive radio in nakagami channels," IEEE Transactions on Vehicular Technology, vol. PP, no. 99, p. 1, 2013.

[13] I. S. Gradshteyn and I. M. Ryzhik, Table of integrals, series, and products, 7th ed. Elsevier/Academic Press, Amsterdam, 2007. 